\title{
O ensino de Endodontia pré-clínica nos cursos de Odontolologia brasileiros
}

\author{
Rafaela Braga Kappler*; Karen Barea de Paula**; Daniela Bazzo Barbisan*; Camila Hélen Grock**; \\ Maria Beatriz Cardoso Ferreira***; Simone Bonato Luisi****; Francisco Montagner****
}

* Cirurgiã-dentista, mestranda m Clínica Odontológica, Programa de Pósgraduação em Odontologia, Universidade Federal do Rio Grande do Sul

** Doutoranda, Programa de Pós-graduação em Odontologia, Faculdade de Odontologia, Universidade Federal do Rio Grande do Sul

*** Professora Titular de Farmacologia, Faculdade de Odontologia, Universidade Federal do Rio Grande do Sul

**** Professor Associado, Departamento de Odontologia Conservadora, Faculdade de Odontologia, Universidade Federal do Rio Grande do Sul

Recebido em 29/11/2018. Aprovado em 21/05/2019.

\begin{abstract}
RESUMO
O objetivo desse estudo foi obter informações quanto ao ensino de Endodontia em atividades préclínicas nos diferentes cursos de graduação em Odontologia no Brasil, além de avaliar o perfil e a distribuição por regiões do país dessas instituições. Foi realizado um estudo observacional transversal do tipo censo, envolvendo 246 instituições de ensino, por meio do acesso aos sítios eletrônicos das respectivas instituições, obtendo-se dados de domínio público. As informações relacionadas às instituições de ensino foram obtidas em base de dados pública, em sítio eletrônico do Conselho Federal de Odontologia (CFO). Foram registradas as seguintes variáveis de interesse: dados sobre a instituição de ensino, a distribuição das disciplinas relacionadas ao ensino de pré-clínica na área de Endodontia, e a carga horária e relacionada com o ensino de Endodontia. Foi realizada análise estatística descritiva dos dados obtidos. A maioria dos cursos ocorrem em instituições privadas, localizadas no Sudeste do país, com maior número de cursos diurnos e apresentando duração mínima de 10 semestres. Em relação à presença de informações relativas à estrutura curricular dos Cursos de Odontologia nas instituições avaliadas, verificou-se que foi possível acesso direto em sítios eletrônicos de 190 instituições $(77,2 \%)$. O Projeto Pedagógico está disponível no sítio eletrônico de 57 cursos $(23,2 \%)$. Observou-se grande diversidade de organização das disciplinas nas diferentes instituições. Constatou-se que há informações limitadas disponíveis para acesso na forma de domínio público, o que dificulta uma análise mais criteriosa acerca dos modelos didáticos e conteúdos abordados pelas instituições. Ainda, observou-se que as informações disponibilizadas sugerem que há abordagens heterogêneas para o ensino da endodontia pré-clínica.
\end{abstract}

Descritores: Endodontia. Ensino. Treinamento. Pré-Clínica. 


\section{INTRODUÇÃO}

A Endodontia é a especialidade da Odontologia que prepara o estudante para o diagnóstico das alterações pulpares e periapicais, com adesão de medidas preventivas e curativas. $\mathrm{O}$ ensino da Endodontia como especialidade em cursos de graduação pressupõe atividades laboratoriais e clínicas ${ }^{1}$. O processo de aquisição das competências e habilidades nesta área da Odontologia pode ser complexo, devido às dificuldades técnicas relacionadas aos procedimentos, às responsabilidades inerentes ao cuidado do paciente e à falta de autoconfiança dos estudantes ${ }^{2}$.

As atividades pré-clínicas compreendem simulações de condições reais e são preparatórias para as atividades clínicas ${ }^{3}$. A área específica de Endodontia, no âmbito de pré-clínica, trabalha conteúdos iniciais para a preparação do aluno. Incluem, na atividade teórica, técnicas de preparo e obturação e condutas a serem realizadas para um tratamento eficaz ${ }^{4}$. Já nas atividades práticas, são ensinadas as técnicas propriamente ditas, incluindo formas de abertura dos dentes, área de acesso ao canal radicular, preparo químico e mecânico, etapa da irrigação, assim como obturação e selamento. É na pré-clínica, também, que o estudante tem contato com os instrumentos utilizados, como limas e brocas específicas para a Endodontia ${ }^{5}$.

Kamaura et al. $(2003)^{6}$ relacionaram o aprendizado laboratorial em Endodontia com sua aplicação nas atividades clínicas durante o período da graduação, no método de ensino integrado. Foram analisados os tratamentos endodônticos realizados por estudantes de graduação da Faculdade de Odontologia da Universidade de São Paulo empregando a documentação radiográfica dos tratamentos executados em manequins no laboratório, e de pacientes atendidos na clínica. Os resultados indicam que existem diferenças do desempenho do estudante nas diferentes tarefas da Endodontia selecionadas para o experimento quando comparadas os grupos dentais tratados e as atividades de laboratório e clínica.

Para Qualtrough e Dummer (2003) ${ }^{8}$, o tempo e a prioridade dispensados ao ensino da Endodontia nos currículos de graduação aumentaram consideravelmente em relação às últimas duas décadas no Reino Unido, reconhecendo a importância desse treinamento ${ }^{7}$. No entanto, os estudantes ainda enfrentam dificuldades, tornando necessária a busca de alternativas curriculares que criem um ambiente mais favorável para a sua formação.

No Brasil, as Diretrizes Curriculares Nacionais não especificam critérios norteadores para o ensino de Endodontia em pré-clínica. Dessa forma, é possível inferir que podem ser propostas diferentes abordagens durante o ensino desta especialidade nas escolas existentes no país?.

Portanto, o presente estudo foi conduzido com o objetivo de se obter informações relativas ao ensino de Endodontia em atividades pré-clínicas, adotados por cursos de Odontologia no Brasil. Além disso, o estudo teve como objetivo avaliar o perfil das instituições de ensino e a distribuição desses cursos em relação às regiões do país.

\section{METODOLOGIA}

O presente estudo foi aprovado pela Comissão de Pesquisa em Odontologia, da Faculdade de Odontologia, Universidade Federal do Rio Grande do Sul (Protocolo número 32422).

Tratou-se de um estudo transversal, do tipo censo, envolvendo consulta a sítios eletrônicos de 246 instituições de ensino brasileiras, públicas ou privadas, cadastradas na página do Conselho Federal de Odontologia (CFO) que oferecem Curso de Graduação em Odontologia (acesso em abril de 2018). As informações obtidas são de domínio público, ou seja, estão disponíveis nos sítios eletrônicos das instituições.

Após consulta ao sítio eletrônico do CFO, os pesquisadores utilizaram a rede mundial de 
computadores para ter acesso aos sítios das respectivas instituições de ensino. Para o registro dos dados, foi utilizado um formulário especificamente elaborado para a realização da pesquisa. Foram consultados os seguintes aspectos: a) dados sobre a natureza da instituição de ensino: pública, privada, comunitária; b) data de atualização do sito eletrônico; c) dados sobre o curso: diurno/noturno, número de semestres; d) dados sobre o ensino de Endodontia pré-clínica: presença/ausência de disciplina de endodontia préclínica, carga horária, semestre em que ocorre; e) dados quanto à disponibilidade de acesso ao projeto pedagógico e/ou à matriz curricular.

Para a análise, considerou-se que os cursos poderiam apresentar "Disciplina de Pré-clínica Integrada" (quando Endodontia é ministrada simultaneamente a outras áreas), "Disciplina de Pré-Clínica de Endodontia" (quando a endodontia é ministrada de forma isolada) e "Disciplina de Endodontia" (quando havia conteúdos de Endodontia laboratorial, clínica ou ambas, não havendo especificação). As demais variáveis analisadas foram o número de semestres de cada disciplina; semestre em que a disciplina é ministrada; número de créditos; carga horária e número de disciplinas.

As respostas foram tabuladas em banco de dados, agrupadas e apresentadas em tabelas. Para análise estatística, foi empregado o pacote estatístico IBM SPSS Statistics v. 21 (IBM Corp. Released 2012. IBM SPSS Statistics for Windows, Version 21.0. Armonk, NY: IBM Corp.). Os dados foram expressos como frequência absoluta ou relativa.

Todos os dados coletados e avaliados são de domínio público, e não estão contidos em banco de dados, que necessitem permissão para acesso. Todas as informações foram publicadas em conjunto, sem que sejam identificadas características particulares de cada instituição.

\section{RESULTADOS}

Foram avaliados 246 sítios eletrônicos de Cursos de Odontologia nas cinco regiões do Brasil. Quanto à data de atualização dos sítios em que foram feitas as coletas, 8,4\% haviam sido atualizados há entre 5 e 10 anos, 35\% há menos de 3 anos, 30,9\% há menos de 2 anos, e 23,6\% no último ano. Em 54,2\% dos sítios não foi possível identificar a sua data de atualização. O perfil das instituições que oferecem o Curso de Odontologia e a distribuição desses cursos, considerando as regiões do país estão representadas no gráfico 1 .

O número total de semestres descrito para cada curso apresentou variações. Prevalecem os cursos com duração de 10 semestres (45,9\%), seguindo-se daqueles de 8 semestres (28\%). Em menor quantidade tem-se 9 semestres $(9,8 \%), 12$ semestres $(1,6 \%)$ e apenas um curso que possui 16 semestres, correspondendo $0,4 \%$, o qual é um curso noturno. Os demais $(14,2 \%)$ não informam esses dados. Informações relativas à estrutura curricular dos cursos foram localizadas em 77,2\% dos sítios eletrônicos. Para o Projeto Pedagógico, os mesmos foram localizados em apenas $23,2 \%$ dos cursos.

A tabela 1 resume os dados obtidos, considerando-se as variáveis estudadas. Constatouse que 6,09\% (15/246) cursos apresentam apenas a "Disciplina de Pré-Clínica Integrada". Esta disciplina tem sido ministrada do primeiro ao oitavo semestre. $\mathrm{O}$ número de créditos variou de 11 a 62 , porém em $47 \%$ dos documentos consultados não havia este dado. A carga horária variou entre $60 \mathrm{e}$ 1015 horas. Esse dado não foi encontrado em $11,7 \%$ dos sítios. É importante salientar que a contagem foi cumulativa, ou seja, se havia mais de uma disciplina de Pré-Clínica Integrada no curso ocorreu somatório de créditos e de horas. Além disso, por se tratar de uma disciplina integrada com outras áreas, não se pode afirmar que essa carga horária é referente aos conteúdos de Endodontia. 
Gráfico 1. Perfil das instituições de ensino brasileiras com cursos de Odontologia e distribuição nas regiões do país $(\mathrm{n}=246)$

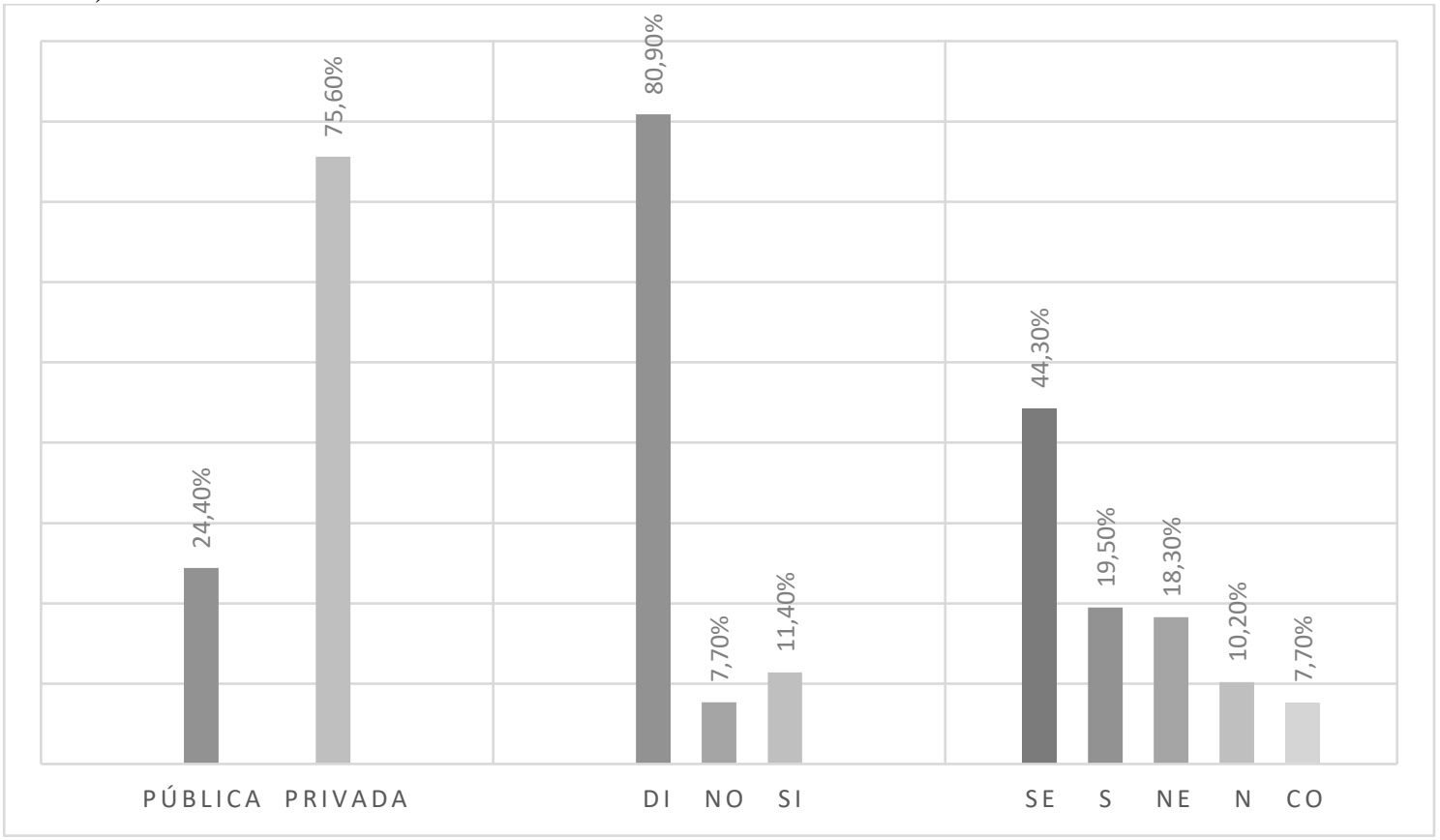

DI: Diurno; NO: Noturno; SI: Sem informação; SE: Sudeste; S: Sul; NE: Nordeste; N: Norte; CO: Centro-oeste.

Tabela 1. Panorama do ensino de atividades pré-clínicas de endodontia em Cursos de Odontologia no Brasil

\begin{tabular}{lcccc}
\hline & PCI & PCE & Endo & NI \\
\hline Frequência & $15(6,09 \%)$ & $40(16,26 \%)$ & $87(35,36 \%)$ & $72(29,26 \%)$ \\
Distribuição no curso* & $1 \rightarrow 8$ & $4 \rightarrow 7$ & $3 \rightarrow 10$ & \\
Número de Semestres & & & & \\
$\quad 1$ semestre & 4 & 44 & 34 & - \\
2 semestres & 2 & 5 & 56 & - \\
3 semestres & 5 & 0 & 12 & - \\
4 semestres & 1 & 0 & 2 & - \\
5 semestres & 1 & 0 & 0 & - \\
$>5$ semestres & 2 & 0 & 0 & - \\
Não informado & 2 & 21 & 15 & - \\
\hline
\end{tabular}

PCI = Pré-clínica Integrada (não apenas endodontia); PCE = Pré-clínica em Endodontia; Endo = Disciplina de Endodontia (consta descrição de atividades em pré-clínica ou clínica); NI = Não informado. * Semestres em que é ministrada a disciplina

A "Disciplina de Pré-clínica de horária, a mesma variou de 51 a 180 horas. Essa Endodontia" é ministrada em 16,26\% (40/246) carga horária é referente apenas aos conteúdos dos cursos. Esta disciplina concentra-se entre o quarto e o sétimo semestres. Em relação à carga ministrados na área de Endodontia.

A "Disciplina de Endodontia” é ministrada 
em 35,36\% cursos (87/246). A distribuição desta disciplina ao longo do curso é bastante heterogênea, podendo ser ministrada do $3^{\circ}$ ao $10^{\circ}$ semestre. Em 25,3\% destas instituições, esta disciplina ocorre no $5^{\circ}$ e no $6^{\circ}$ semestres. Quanto ao número de "Disciplinas de Endodontia" ao longo do curso, verifica-se que varia entre 1 e 4 . Considerando-se a carga horária, a mesma variou entre 56 e 280 horas. Não se pode afirmar se essa carga horária condiz com os conteúdos de endodontia pré-clínica ou clínica, já que essa disciplina pode abordar ambos.

Em nenhuma das instituições avaliadas as Disciplinas de Pré-Clínica Integrada e PréClínica em Endodontia são ministradas em um mesmo curso. Em 2 instituições $(0,81 \%)$ a Disciplina de Pré-Clínica Integrada e a Disciplina de Endodontia estão presentes no mesmo curso. Em 30 instituições (12,19\%) são ministradas ambas as Disciplina de Pré-Clínica em Endodontia e a Disciplina de Endodontia.

\section{DISCUSSÃO}

Sabe-se que o treinamento da Endodontia pré-clínica é fundamental, já que este momento oferece aos estudantes a oportunidade de obter e consolidar o conhecimento antes de realizar tratamentos em pacientes ${ }^{2,10}$. Embora exista um número importante de cursos de graduação em Odontologia no Brasil, observa-se uma escassez de estudos que apresentem informações relativas ao treinamento pré-clínico em Endodontia, em relação ao qual possivelmente existem divergências pouco conhecidas. A percepção dessas diversidades poderia contribuir para o reconhecimento de estratégias que favoreçam o processo de ensino e aprendizagem. Portanto, o presente estudo foi realizado com o objetivo de buscar informações referentes ao ensino de endodontia em atividades pré-clínica nos cursos de graduação em Odontologia do Brasil.

A metodologia utilizada foi de caráter unilateral, ou seja, houve busca ativa do pesquisador em endereço eletrônico, sem solicitação de informações adicionais a qualquer instituição. Assim, as limitações deste trabalho estão baseadas em duas grandes vertentes: a escassez de informações básicas nos sítios de busca e a data de atualização dos endereços eletrônicos. A primeira, claramente observada no número pequeno de informações completas disponíveis nas disciplinas, bem como na disponibilidade da matriz curricular e do projeto pedagógico. A segunda, mostrando que há sítios que não atualizam seus dados com frequência, o que impossibilita o entendimento sobre a realidade atual do curso. Além disso, outra limitação encontrada é o fato de não se conhecer, em muitos cursos a descrição de cada disciplina no plano de ensino, não havendo segurança para interpretar qual o conteúdo que de fato é trabalhado.

É importante que informações referentes ao curso sejam disponibilizadas em sítios de livre acesso. A divulgação da matriz curricular do curso estava presente em 77,2\% dos sítios eletrônicos. No entanto, o projeto pedagógico é divulgado por apenas $23,2 \%$ das instituições. $\mathrm{O}$ projeto pedagógico é um referencial teóricometodológico que mostra o funcionamento do curso e o rumo traçado para o ensino, além de orientar na busca para realização de pesquisas, na escolha profissional e na dinâmica entre diferentes escolas. É um documento obrigatório para todas as instituições e tem como característica ser dinâmico, isto é, não tem um momento que se encerra, está em constante processo de construção ${ }^{11}$. Apesar da importância do projeto pedagógico, dados da literatura revelam que há um desconhecimento dos discentes sobre as regulamentações que orientam o ensino superior ${ }^{12}$.

Os cursos diurnos constituem 80,9\% da amostra, enquanto que os cursos noturnos $7,7 \%$. 
A presença de cursos de graduação em Odontologia no período noturno têm se tornado um instrumento de inclusão social ${ }^{13}$. No mundo atual, sabe-se da necessidade de conciliar trabalho e estudo e, como o curso apresenta carga horária extensa, é interessante oferecer aos estudantes que trabalham a oportunidade de frequentar a faculdade no turno da noite.

Há um predomínio de cursos de Odontologia que são ofertados por instituições privadas $(75,6 \%)$. Nesse estudo, constatou-se a seguinte distribuição em relação às regiões do país: Norte $(10,2 \%)$, Nordeste $(18,3 \%)$, CentroOeste $(7,7 \%)$, Sudeste $(44,3 \%)$ e Sul $(19,5 \%)$. É evidente a maior concentração de cursos na região Sudeste, seguida do Sul e Nordeste. Sabese que a concentração dos cirurgiões-dentistas após a formação segue o mesmo padrão. Três quartos deles estão concentrados no Sul e Sudeste do país, distribuição esta que se assemelha à distribuição da participação percentual das grandes regiões no PIB $^{14}$.

As Diretrizes Curriculares Nacionais (DCN) estabelecem que na estrutura do Curso de Graduação em Odontologia devem existir três ciclos: básico, pré-profissional e profissional. As atividades de treinamento pré-clínico conectam os ciclos inicial e final, e têm por finalidade aproximar o conhecimento básico da sua utilização clínica. Na presente pesquisa, verificase que a maioria das escolas inicia o ensino préclínico de Endodontia a partir do $4^{\circ}$ semestre, após a realização das disciplinas básicas. Uma abordagem diferente foi relatada em estudo desenvolvido no Reino Unido, em que a instrução pré-clínica de Endodontia é iniciada durante o primeiro ano clínico em todas as escolas que participaram da pesquisa ${ }^{7}$. Em estudo realizado em 2015 nas ilhas Fiji, indicou-se que módulos de atividades pré-clínicas são incorporados no currículo principalmente nos dois primeiros anos do curso ${ }^{15}$. Demonstra-se assim uma abordagem bastante semelhante àquela adotada nos cursos brasileiros analisados neste estudo.

Os estudantes relatam com frequência a necessidade de treinamento pré-clínico intenso, pois o tempo limitado no treinamento pré-clínico e clínico em Endodontia pode resultar em baixa auto-confiança durante a prática de atendimento a pacientes, indicando insatisfação e insegurança do estudante ${ }^{16}$. Em estudo realizado nas Filipinas, constatou que o número limitado de horas atribuído pela maioria das escolas privadas precisa de reavaliação, já que o Conselho de Educação Odontológica indica que os cursos de graduação devem fornecer pelo menos 4 horas semanais de ensino de Endodontia pré-clínica ${ }^{17}$.

Pode-se dizer, dessa maneira, que os resultados deste estudo devem ser vistos com cautela, especialmente porque muitos sítios de consulta não haviam sido atualizados com frequência. Quando presentes, as informações muitas vezes não eram precisas ou detalhadas de forma suficiente. Ainda, sabe-se que as atividades pré-clínicas são trabalhadas ao longo do curso. Porém, muitas vezes, não são apresentadas de forma objetiva as informações quanto a sua inserção no currículo, carga horária, número de créditos e súmula da disciplina.

Observa-se que não há um padrão que caracteriza o oferecimento das atividades de préclínica nos cursos de Odontologia no Brasil. Existem Diretrizes Curriculares Nacionais do Curso de Graduação em Odontologia, que definem os princípios, fundamentos, condições e procedimentos da formação de Cirurgiões Dentistas, estabelecidas pela Câmara de Educação Superior do Conselho Nacional de Educação, para aplicação em âmbito nacional na organização, desenvolvimento e avaliação dos projetos pedagógicos dos Cursos de Graduação em Odontologia das Instituições do Sistema de Ensino Superior ${ }^{9}$. O documento aborda as áreas 
básicas exigidas, porém não discute de maneira específica os conteúdos que devem ser ofertados em cada disciplina e números mínimos a serem respeitados, como carga horária para cada área, número de créditos, quantidade de semestres, entre outras regulamentações. Estudo feito em 2008 indica que a uniformização dos currículos de endodontia não tem como objetivo fornecer instruções definitivas e padronização dos métodos de ensino, uma vez que os modos inovadores dos currículos para o ensino em endodontia devem ser encorajados ${ }^{18}$. Porém, sugestões incorporadas nas DCN ou consensos fornecidos por sociedades de especialistas poderiam ser empregados para que existissem sugestões de requisitos mínimos a serem utilizados por cada instituição, servindo como um roteiro para garantir um melhor nível de exigência, assegurando um modelo de ensino mais igualitário e buscando a formação de um profissional capacitado científica e tecnicamente.

\section{CONCLUSÃO}

Conforme os resultados demonstrados no presente estudo, foi identificado que, frequentemente, as informações referentes ao ensino de Endodontia pré-clínica nos cursos de Odontologia do Brasil não estão disponíveis nos endereços eletrônicos das instituições para domínio público. Houve, consequentemente, dificuldade em se obter informações mais representativas referentes à súmula de disciplinas, à carga horária e ao número de créditos. Assim, torna-se difícil uma análise mais aprofundada sobre o currículo dos cursos e também sobre os modelos didáticos adotados. Considerando-se o perfil das instituições que abrigam Cursos de Odontologia no país, foi possível observar que a maioria é privada, está concentrada na região Sudeste do país e é ofertada no período diurno. Observa-se uma pluralidade na estruturação do ensino de
Endodontia pré-clínica no Brasil, o que parece respeitar o pensamento e a forma de condução do processo de ensino e aprendizagem da especialidade, adotados em cada escola, pelos diferentes grupos de professores. Caso houvesse maiores informações disponíveis para acesso público, seria possível caracterizar de forma mais precisa as condutas adotadas a fim de que se estabeleça uma troca de experiências entre as instituições, tendo como objetivo contribuir para a formação do estudante de graduação.

\section{ABSTRACT \\ Preclinical Endodontics teaching in Brazilian Dentistry courses}

This study aimed to achieve information concerning the teaching of Endodontics in preclinical activities in different undergraduate courses in Dentistry in Brazil, and analyzed the profile and distribution of these institutions across the country regions. This was a crosssectional observational census study, involving 246 teaching institutions, by accessing the electronic websites of institutions, achieving public domain data. The information related to the teaching institutions were obtained from a public database, on the electronic website of the Brazilian Federal Dental Council (CFO). The following variables of interest were recorded: data on the teaching institution, distribution of disciplines related to preclinical teaching in the field of Endodontics, and workload related to the teaching of Endodontics. Data were submitted to descriptive statistical analysis. Most courses are offered in private institutions, located in the Southeast region of the country, with greater number of daytime courses presenting minimum duration of 10 semesters. Concerning the presence of information related to the curriculum of Dentistry courses in the institutions analyzed, direct access was possible to the electronic websites of 190 institutions (77.2\%). The Pedagogical Project was available on the electronic website of 57 courses $(23.2 \%)$. There was great diversity of organization of disciplines in the different institutions. It was observed that 
there is limited information available for access as public domain, which impairs a more careful analysis of the didactic models and contents addressed by the institutions. Also, the information available suggest heterogeneous approaches for the preclinical Endodontics teaching.

Descriptors: Endodontics. Teaching. Training. Preclinical.

\section{REFERÊNCIAS}

1. Andriola WB, Andriola CG. Assessment of educational quality of the Faculty of Education (FACED) of the Federal University of Ceará (UFC). Ensaio: Aval Pol Públ Educ. 2009;17(62):153-68.

2. Seijo MOS, Ferreira EF, Sobrinho APR, Paiva SM, Martins RC. Learning Experience in Endodontics: Brazilian students' perceptions. J Dent Educ. 2013;77(5):64855.

3. European Society of Endodontology. Quality guidelines for endodontic treatment: consensus report of the European Society of Endodontology. Int Endod J. 2006;39(12):921-30.

4. de Moor R, Hülsmann M, Kirkevang L-L, Tanalp J, Whitworth J. Undergraduate curriculum guidelines for endodontology. Int Endod J. 2013;46(12):1105-14.

5. FO-UFRGS. Projeto Pedagógico do Currículo Diurno do Curo de Odontologia. 2014 [Acesso em 10 jun. 2015]. Disponível em: http://www.ufrgs.br/odontologia/ensi no/odonto/graduacao/projeto-pedagogicodo-curso-diurno/view

6. Kamaura D, Carvalho G, Lage-Marques J, Antoniazzi J. Avaliação do desempenho dos alunos de graduação durante a prática da técnica endodôntica. Rev ABENO. 2003;3(1):33-40.

7. Qualtrough AJE, Dummer PMH. Undergraduate endodontic teaching in the
United Kingdom: an update. Int Endod J. 2003;30(4):234-9.

8. Mirza MB. Difficulties encountered during transition from preclinical to clinical endodontics among Salman bin Abdul Aziz University Dental students. J Int Oral Health. 2015;7(Suppl 1):22-7.

9. Brasil. Conselho Nacional de Educação. Câmara de Educação Superior. Resolução CNE/CES 3/2002, de 19 de fevereiro de 2002. Institui as Diretrizes Curriculares Nacionais do Curso de Graduação em Odontologia. Seç. 1 mar 4, 2002.

10. Tchorz JP, Brandl M, Ganter PA, Karygianni L, Polydorou O, Vach K, et al. Pre-clinical endodontic training with artificial instead of extracted human teeth: does the type of exercise have an influence on clinical endodontic outcomes? Int Endod J. 2015;48(9):888-93.

11. Moimaz SAS, Fadel CB, Saliba A. Projeto pedagógico e estrutura curricular de um curso de Odontologia: análise crítica fundamentada na percepção acadêmica. Rev ABENO. 2010; 10(2):35-40.

12. Ferraz MÂAL, Falcão CAM, Pereira RM de S, Pinheiro COB, Nascimento EPA do, Moura MEB. Projeto pedagógico do Curso de Odontologia: conhecimento discente. Rev ABENO. 2015;15(2):47-55.

13. Filho AT, Nery ACB. Ensino superior noturno no Brasil: história, atores e políticas. Rev Bras Pol Adm Educ. [Acesso em 11 jun. 2018]. Disponível em: http://www.seer. ufrgs.br/index.php/rbpae/article/view/19327

14. Moritta MC, Haddad AE, Araújo ME. Perfil atual e tendências do cirurgião-dentista brasileiro [Internet]. Perfil atual e tendências do cirurgião-dentista brasileiro. 2010 [Acesso em 11 jun. 2018]. Disponível em: http://cfo.org.br/wp-content/uploads/2010/ 04/PERFIL_CD_BR_web.pdf 
15. Devi AA, Abbott PV. An overview of the endodontic curriculum in Fiji from 2009 to 2013. Austr Endod J. 41(3):111-6.

16. Grock C, Francisco M, Luciana L, Maria Beatriz F, Vanessa F, Lisiane B, et al. Experiências relacionadas à execução de tratamentos endodônticos de urgência e níveis de ansiedade, qualidade do sono e qualidade de vida em alunos de graduação em Odontologia [Dissertação de mestrado]. Universidade Federal do Rio Grande do Sul; 2016.
17. Cruz EV, Jimena ME, Puzon EG, Iwaku M. Endodontic teaching in Philippine dental schools. Int Endod J. 2000;33(5):427-34.

18. Sonntag D, Bärwald R, Hülsmann $M$, Stachniss V. Pre-clinical endodontics: a survey amongst German dental schools. Int Endod J. 2008;41(10):863-8.

\section{Correspondência para:}

Prof Dr Francisco Montagner

e-mail: francisco.montagner@ufrgs.br

Universidade Federal do Rio Grande do Sul

Faculdade de Odontologia

Rua Ramiro Barcelos, 2492 Santana

90035-003 Porto Alegre/RS 